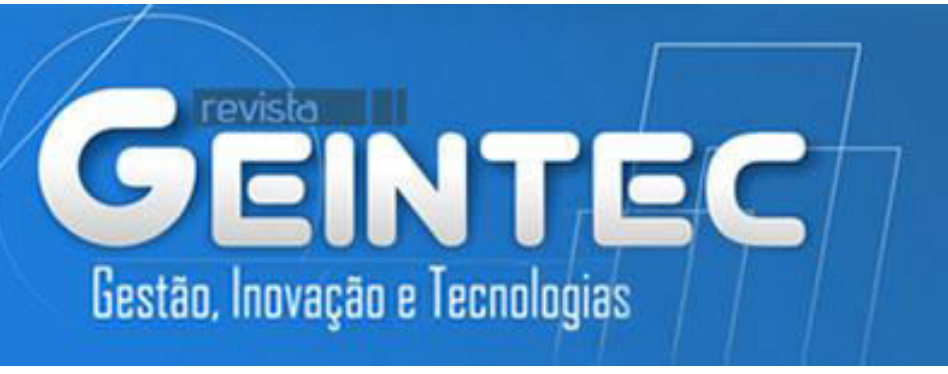

\title{
GESTÃO DE INOVAÇÃO: COMPARAÇÃO DE DUAS REALIDADES DISTINTAS - UNIVERSIDADE ESTADUAL DE SANTA CRUZ (ILHÉUS, BAHIA) E UNIVERSIDADE DE LA ROCHELLE (FRANÇA)
}

\section{INNOVATION MANAGEMENT: COMPARISON BETWEEN TWO DISTINCT REALITIES - UNIVERSIDADE ESTADUAL DE SANTA CRUZ (ILHÉUS, BAHIA) AND UNIVERSIDADE DE LA ROCHELLE (FRANCE)}

\author{
Fabiana Carneiro Silva de Holanda ${ }^{1}$; Gesil Sampaio Amarante Segundo ${ }^{2}$; Mickaël Coustaty ${ }^{3}$ Eric de \\ Almeida Monteiro ${ }^{4}$ \\ ${ }^{1}$ Universidade Estadual de Santa Cruz - UESC - Ilhéus/BA - Brasil \\ fabianahlanda2010@ hotmail.com \\ ${ }^{2}$ Universidade Estadual de Santa Cruz - UESC - Ilhéus/BA - Brasil \\ gesil.amarante@gmail.com \\ ${ }^{3}$ Université de La Rochelle - ULR - La Rochelle - France \\ m.coustaty@valconum.fr \\ ${ }^{4}$ Université de La Rochelle - ULR - La Rochelle - France \\ eric.monteiro@univ-lr.fr
}

\begin{abstract}
Resumo
O presente trabalho consiste em apresentar a realidade da Universidade Estadual de Santa Cruz (UESC) (Ilhéus/Bahia) e da Universidade de La Rochelle (ULR) (França) no que tange às atividades de gestão da inovação, propriedade intelectual (PI) e transferência de tecnologia (TT) executadas em cada universidade. São apresentadas as estruturas universitárias, estrutura dos Núcleos de Inovação Tecnológica, legislação vigente sobre o tema inovação de cada país, patentes depositadas e os casos de transferência de tecnologia. O objetivo é comparar as atividades desenvolvidas nas distintas realidades e buscar, por intermédio das melhores práticas, uma possibilidade de adaptação destas práticas ao caso brasileiro. Os resultados foram alcançados através do levantamento bibliográfico, sobretudo referente à legislação vigente sobre inovação e consulta aos bancos de dados das referidas universidades, além de entrevista feita com o diretor do ULR Valor, Prof. Jean-Marc Wallet. Percebeu-se que as universidades possuem quase a mesma idade e compartilham dos mesmos esforços para desenvolver as atividades de pesquisa, ensino e extensão. No que tange aos processos de gestão da inovação, PI e TT, a ULR possui uma estrutura diferente da UESC, e goza, por intermédio de dispositivos legais, de maior flexibilidade nas suas ações, o que a confere maior êxito e rapidez nos processos de transferência de tecnologia.
\end{abstract}


Palavras-chave: Gestão da Inovação, Transferência de Tecnologia, Núcleo de Inovação Tecnológica, Propriedade Intelectual.

\begin{abstract}
This paper aims to present the reality of the Universidade Estadual de Santa Cruz (UESC) (Ilhéus / Brazil) and the Universidade de La Rochelle (ULR) (France) in relation to the activities of innovation management, intellectual property (IP) and technology transfer (TT) performed at each university. The structure of the universities and of their Technology Transfer Offices, the legislation on innovation of each country, patents deposited and cases of technology transfer are presented. The objective is to compare the activities developed in the different realities and to seek, through best practices, a possibility to adapt those practices to the Brazilian case. The data were derived from the revision of the literature especially regarding the legislation on innovation, from the databases of the universities, and from an interview with the director of the ULR value, Prof. JeanMarc Wallet. It was noticed that the universities have almost the same age and share the same efforts to develop research, teaching and extension activities. The ULR has a structure different from UESC regarding to the management of innovation processes, IP and TT, which allows, by means of legal provisions, greater flexibility in their actions, resulting in more successful and agile processes of technology transfer.
\end{abstract}

Key-words: Innovation Management, Technology Transfer, Technology Transfer Offices, Intellectual Property.

\title{
1. Introdução
}

O tema inovação tecnológica vem tomando lugar de destaque na agenda econômica de diversos países. Considerada como elemento-chave propulsor para o crescimento econômico, a inovação tem se tornado o fator diferencial para a obtenção de vantagem competitiva num mercado cada vez mais global e competitivo.

As discussões e debates acerca do tema possuem diversas vezes o objetivo de estabelecer políticas públicas para promover uma relação de troca entre os institutos de pesquisa públicos e o setor privado, sobretudo no que tange ao desenvolvimento de pesquisa em conjunto e transferência de tecnologias.

Com a consciência de que o estabelecimento da cooperação U-E é uma das mais importantes ferramentas para promoção da inovação e desenvolvimento econômico de um país, o movimento para promoção dessas parcerias vem crescendo a cada ano no mundo todo.

É comum nos debates, discussões e literatura científica a apresentação de dados estatísticos e de casos de boas práticas de outras realidades, principalmente no que se refere aos assuntos de proteção da propriedade intelectual e de transferência de tecnologia de países como EUA, Coreia do Sul, Alemanha e Japão, entre outros. Autores nacionais como Vargas (1997), Andreassi (1999), Takahashi e Sacomano (2002); Tigre (2006), Dias (2007), Balbinot e Marques (2009), Garnica e 
Torkomian (2009); Santana e Porto (2009); Costa e Porto (2011) já citaram em seus trabalhos diferentes realidades.

Ainda que em concordância com Dias (2007), que alerta para o alcance de resultados insatisfatórios no quesito inovação em razão da apropriação indiscriminada de modelos estrangeiros, ou ainda com Malkin (2005), que aponta para o perigo de mascarar realidades ao comparar as bases de indicadores internacionais e de diferentes sistemas de pesquisa e inovação, sobretudo no que tange aos indicadores de performance de diferentes países, que possuem entre eles diferenças nas questões de políticas públicas, financiamento, especificidades institucionais e características estruturais, acredita-se que através da comparação entre diferentes realidades, e resguardando as diferenças já mencionadas, seja possível encontrar soluções ou estabelecer novos paradigmas para as estratégias de inovação de um determinado organismo, seja ele público ou privado.

Nesse sentido, é traçado o objetivo do presente trabalho. Pretendeu-se demonstrar a realidade das distintas universidades, Universidade Estadual de Santa Cruz, Ilhéus/Bahia e Universidade de La Rochelle, França, no que se refere aos processos gestão da inovação, transferência de tecnologia e propriedade intelectual e comparar as práticas estabelecidas em cada uma delas, apontando as semelhanças e diferenças, possibilitando a apropriação para a realidade brasileira das boas práticas encontradas.

O trabalho divide-se em introdução, metodologia, descrição dos aspectos relacionados tanto à UESC quanto à ULR referentes à estrutura universitária, estrutura do Núcleo de Inovação Tecnológica, legislação vigente para inovação, contratos de transferência de tecnologia, patentes e exemplos de casos de transferência de tecnologia, discussão e conclusão.

\section{Metodologia}

A construção do trabalho foi baseada em revisão bibliográfica, tanto da literatura nacional quanto da literatura francesa sobre o tema inovação, PI e TT, em consulta de documentos das universidades UESC e ULR, além de entrevista feita no dia 15 de março de 2013 com o Prof. JeanMarc Wallet, diretor do organismo ULR Valor, sobre o processo de gestão da inovação, propriedade intelectual e transferência de tecnologia da Universidade de La Rochelle.

\section{Universidade Estadual de Santa Cruz}

A Universidade Estadual de Santa Cruz (UESC) foi criada em 1991 a partir da estadualização da FESPI (Federação das Escolas Superiores de Ilhéus e Itabuna) pelo então 
Governador do Estado que a incorporou ao quadro das Universidades públicas da Bahia (UESC, 2013).

A área de abrangência de atuação da UESC corresponde à região sul da Bahia, composta pelas regiões econômicas do Litoral Sul, com 53 municípios, e do Extremo Sul, com 21 municípios. A UESC pode ser considerada a instituição mais estruturada no que se refere a ensino e pesquisa da região sul da Bahia.

Os últimos dados apresentados pela assessoria de planejamento (ASPLAN, 2012) apontam que a Universidade possui:

- 33 cursos presenciais, dos quais 11 são de licenciatura e 22 de bacharelado, e 4 cursos de Educação à Distância;

- 7.853 alunos de graduação divididos entre 6.830 presenciais e 1.023 à distancia;

- 794 professores, sendo 386 doutores, 319 mestres, 77 especialistas e 12 graduados;

- 28 cursos de Pós-Graduação, sendo 19 Stricto sensu (4 doutorados e 15 mestrados) e 9 Lato sensu, com o total de 767 alunos matriculados;

- A estrutura de laboratórios e centro de pesquisa é formada por 30 laboratórios, divididos nas áreas de conhecimento: exatas, saúde e humanas.

No que tange aos aspectos de Propriedade Intelectual, a UESC institucionalizou o Núcleo de Inovação Tecnológica em 2009 e formalizou sua Política de Inovação em 2010 através da resolução CONSU 10/2010.

Criado em 2009 através da resolução CONSU (Conselho Superior) 05/2009, o NIT é uma instância ligada à Reitoria da UESC, que tem por objetivo promover a inovação e a adequada proteção das invenções geradas nos âmbitos interno e externo da UESC e a sua transferência ao setor produtivo, visando integrá-las à comunidade e contribuir para o desenvolvimento (CONSU, 2009).

Atualmente o NIT possui seu quadro de funcionários formado por cinco profissionais contatados por regime de bolsa e um funcionário da universidade. Todos estão sob a coordenação do Prof. Dr. Gesil Sampaio Amarante Segundo.

Segundo a resolução 05/2009, o NIT apresenta seu organograma dividido em três comissões e para cada uma existe um professor da instituição responsável por suas atividades:

I. Comissão de Propriedade Intelectual;

II. Comissão de Informações Tecnológicas;

III. Comissão de Transferência de Tecnologia.

Integrante da REDENIT-Nordeste e do Fórum Nacional de Gestores de Inovação e Transferência de Tecnologia (FORTEC), o NIT da UESC tem se mostrado bastante atuante, desde 
sua criação, na promoção ou apoio a eventos científicos ou de extensão que tenham como objetivo a disseminação da cultura da Propriedade Intelectual (PI) e Transferência de Tecnologia (TT).

Além da promoção e apoio a eventos, o NIT desempenha um importante papel de auxiliar os pesquisadores da universidade ou inventores independentes na proteção da PI. Antes da criação do NIT, a UESC possuía duas patentes em parceria com outras universidades (PI 06033544-2 A2, PI 0801447-7 A2). Atualmente, ela possui um total de nove patentes depositadas cadastradas no banco de dados do INPI: PI 1012479-9 A2, PI 1101532-2 A2, PI 1106477-3 A2, BR 1020120084210 A2, BR 1020120084228 A2, BR 1020120084236 A2 e BR 1020120218380 A2 (INPI, 2013).

No que se refere à legislação vigente no Brasil a qual está vinculada a UESC, no que tange aos assuntos diretamente ligados à PI e TT, existem a Lei N 11.174 de 09 de Dezembro de 2008 (Lei da Inovação da Bahia), que dispõe sobre incentivos à inovação e à pesquisa científica e tecnológica em ambiente produtivo no Estado da Bahia e dá outras providências (SECTI, 2008), e a Lei 10.973 de 2 de Dezembro de 2004 (Lei federal de Inovação), que foi promulgada com os objetivos de criar um ambiente propício a parcerias estratégicas entre as universidades, institutos tecnológicos e empresas; incentivar a participação de institutos de ciência e tecnologia no processo de inovação; e estimular a inovação na empresa — busca potencializar a aplicação de recursos em pesquisa e desenvolvimento, tanto nas instituições públicas quanto nas empresas, contribuindo para aumentar o desenvolvimento e a competitividade dos produtos brasileiros (MATIAS-PEREIRA E KRUGLIANSKAS, 2005).

Ainda no que se refere às normas legais para as atividades de PI e TT, o NIT da UESC possui suas atribuições estipuladas pela resolução CONSU 05/2009, além de seguir as orientações previstas na Política Institucional da Inovação Tecnológica da UESC.

Em relação a contratos de TT, a UESC ainda não celebrou nenhum contrato de licenciamento de patente, apenas convênios para pesquisa em parceria.

\section{Universidade de La Rochelle}

A Universidade de La Rochelle foi criada em 20 de Janeiro de 1993. Fruto do Relatório Regional de Planificação e Desenvolvimento de Formação Superior lançado em Novembro de 1990 que apontava a necessidade da criação de uma segunda universidade na Região de PoitouCharentes, a universidade ocupa atualmente um lugar de destaque entre as universidades da região (ULR, 2013).

A área de abrangência de atuação da ULR corresponde ao departamento de CharenteMaritime como também toda a região de Poitou-Charentes, haja vista que $45 \%$ do total de alunos são originários do departamento de Charente-Maritime, 60\% dos alunos matriculados na graduação 
são da região de Poitou-Charentes, e 56\% dos mestrandos são de outra região francesa ou são estrangeiros (ULR, 2013).

A Universidade possui, de acordo com os últimos dados publicados (ULR, 2013):

- 3 unidades de formação de pesquisa: Direito, Ciência Política e Gestão; Ciência Fundamental e Ciência das Engenharias, e Letras, Línguas, Artes e Ciências Humanas;

- 69 diplomas nacionais divididos em: 2 diplomas de acesso aos estudos universitários, 5 diplomas universitários de tecnologia, 13 licenciaturas, 17 bacharelados;

- 7.417 alunos matriculados;

- 503 professores e professores-pesquisadores;

- 38 cursos de pós-graduação sendo 32 mestrados e 6 doutorados;

- 15 unidades laboratoriais de pesquisa;

- 1 Fundação Universitária;

- 1 Filial dedicada a valorização da pesquisa - ULR Valor.

No que tange aos assuntos referentes à Propriedade Intelectual e Transferência de tecnologia, atualmente a ULR possui um organismo formalizado e em funcionamento, ULR Valor, que foi criado em 2003 e representa uma das primeiras filiais universitárias dedicadas à valorização da pesquisa (ULR VALOR, 2013).

Sob o regime legal de sociedade de direito privado com capital social de 70.000 euros, configurada como uma SAS (Société par actions simplifiée), a ULR valor trata-se de uma sociedade de economia mista em que a ULR é acionista majoritária, com $62 \%$ das ações, seguida das instituições de crédito francesas: Caisse des Dépôts et Consignations (16\%), Caisse fédérale du Crédit Mutuel Océan (11\%), Crédit Agricole Mutuel 17-79 (5,5\%) e Banque Populaire Centre Atlantique (5,5\%) (ULR Valor, 2013)

Esse organismo possui a função de acompanhar os projetos de pesquisa e de prestação de serviços, conduzir as negociações e preparações dos contratos para transferência de tecnologia, elaborar os estudos de viabilidade econômica para as tecnologias da universidade, buscar novos parceiros para projetos em cooperação, auxiliar a proteção da propriedade intelectual e acompanhar a criação de novas empresas (URL Valor, 2013).

A equipe de trabalho do ULR Valor é formada apenas pelo diretor e uma secretária, todos contratados da universidade.

Ainda sobre o mesmo tema, segundo o contrato plurianual de desenvolvimento (2013), a ULR possui o planejamento de reorganizar seu sistema de valorização da pesquisa. Para tanto, a ULR participará da criação de uma Sociedade de Aceleração de Transferência de Tecnologia, SATT 
Grand Centre, que acompanhará os projetos de pesquisa em parceria além de desenvolver, juntamente com o ULR Valor, as atividades de gestão da PI, TT, consultoria e prestação de serviço ao setor empresarial (ULR, 2013).

De acordo com Prof. Jean-Marc Wallet, diretor da filial ULR Valor, a SATT terá uma grande importância no processo de gestão da PI e TT. Ela terá como objetivos principais a detecção de projetos em desenvolvimento dentro da universidade, o financiamento, através do fundo de maturação, de projetos de forte potencial econômico e tecnológico, de depósito de patentes, de construção de protótipos, de estudo de mercado ou de marketing e ainda, ela auxiliará os processos de licenciamento de patentes.

Além dos organismos citados, a ULR conta com a atividade de uma fundação universitária criada em 2009 com o intuito de promover mais autonomia à universidade no estabelecimento das parcerias com empresas. A Fundação Universidade de La Rochelle é fruto da concessão de direito possibilitada pela Lei de Liberdade e Responsabilidade das Universidades de 11 de Agosto de 2007 e, segundo Prof. Jean-Marc Wallet, atualmente exerce uma importante função para viabilizar a captação de recursos de financiamento externo às atividades da universidade, sobretudo as de pesquisa.

Ainda segundo Prof. Jean-Marc Wallet, a fundação da ULR tem alcançado interessantes parcerias entre a universidade e o setor privado para a construção de projetos, principalmente de P\&D. Um caso que inicialmente foi estabelecido como um projeto de parceria para pesquisa e que posteriormente se formalizou como associação entre a ULR e os demais entes privados foi o Projeto Valconum.

O Projeto Valconum nasceu da colaboração inicial da APROGED (Associação de profissionais da economia digital) com o laboratório L3i da ULR, da equipe pedagógica do mestrado ICONE da ULR com o objetivo de desenvolver projetos de P\&D em parceria (VALCONUM, 2013). Este projeto representa um dos casos ocorridos na ULR para TT através de elaboração de convênios para pesquisa.

Com a participação de novas universidades e empresas ao projeto e a necessidade de expandir as propostas de projetos de pesquisa em parceria, a ULR juntamente com os demais grupos envolvidos no projeto Valconum, tanto da iniciativa privada quanto pública, decidiram transformar o projeto em uma associação sem fins lucrativos.

Baseada na lei francesa de 1901 que trata dos contratos de associação, o novo modelo criado pela universidade tinha o objetivo de propor uma forma diferente de possibilitar a transferência direta de tecnologia através de projetos desenvolvidos pela ULR e financiados, em parte ou em totalidade, pelas empresas associadas. Atualmente, Valconum é formado por sete 
empresas privadas, dois organismos do governo e cinco laboratórios de pesquisa das universidades de La Rochelle, Tour, Poitiers e Lyon (VALCONUM, 2013).

Muito embora o objetivo principal seja facilitar a gestão de PI e promover uma TT de forma mais rápida e direta, o Valconum ainda não possui uma política de inovação formalizada, cabendo a análise de PI e TT caso a caso.

No que concerne à legislação sobre o tema pesquisa e inovação, de acordo com Uzunidis (2001, p.167), a França, desde os anos 60, vem desenvolvendo seu modelo jurídico de inovação de forma a possibilitar ligações entre a pesquisa pública e as empresas. Segundo Mérindol (2005, p.108), as leis de 1968 e 1984 permitiram às universidades de estabelecer contratos, depositar patentes em seus nomes, criar filiais privadas, de formular contratos com o Estado, com a sociedade e com empresas.

Ainda sobre a legislação, a lei francesa que versa sobre Inovação e Pesquisa é a lei de 12 de Julho de 1999. Segundo Uzunidis (2001, p.168), essa lei tem como objetivo desenvolver e promover a inovação através de quatro vias: 1. Aumentando a troca entre universidades e empresas, 2. Possibilitando a criação de institutos de valorização da pesquisa, 3. Possibilitando ao pesquisador transformar-se em empreendedor e 4. Estipulando vantagens fiscais e jurídicas às empresas inovadoras.

\section{Similaridades e Diferenças}

Tanto a UESC quanto a ULR são universidades jovens. A ULR no ano de 2013 celebra 20 anos de existência, enquanto a UESC completa 22. Ambas as universidades desenvolvem suas atividades no âmbito de ensino, pesquisa e extensão. Apesar de possuírem estruturas curriculares diferentes, elas apresentam quase o mesmo número de cursos de graduação e um número muito próximo de alunos, além de exercerem um importante papel no desenvolvimento sócio econômico nas suas regiões de abrangência.

Ambas as universidades possuem a preocupação de desenvolver estratégias e soluções para promover o estabelecimento de parcerias entre o setor público e privado, sobretudo para o desenvolvimento de pesquisas em conjunto, ou mesmo, a transferência de tecnologias já criadas por elas.

Contudo, apesar das similaridades, as primeiras diferenças são percebidas na própria estrutura universitária no que diz respeito aos processos de gestão de PI e TT. Enquanto a UESC possui um NIT vinculado à Reitoria da universidade e atrelado às questões burocráticas de instituições públicas, a ULR possui, como equivalente ao NIT, uma empresa de sociedade mista de 
direito privado o que possibilita uma maior flexibilidade e agilidade na execução das suas tarefas e funções.

Além disso, a ULR possui uma fundação que confere à universidade um canal para captação de recurso e investimento direto em diversas áreas do conhecimento, sobretudo na pesquisa, o que torna o desenvolvimento de projetos em parceria entre U-E um processo mais fácil e fluido.

Embora ambas as universidades sejam vinculadas à estrutura pública, e sejam universidades públicas, os dispositivos legais que regem tantos as universidades quanto suas atividades de gestão de PI e TT são, em alguns importantes aspectos, diferentes.

A ULR possui uma maior autonomia no que concerne as suas ações e decisões. Segundo Mérindol (2005, p. 109), a autonomia científica, pedagógica e de gestão (criação de diploma, organização interna, contratação de mão de obra, criação de filiais privadas) foram possibilitadas e expandidas pelas medidas legislativas.

De acordo com Viginier (2002, p. 111), as políticas legislativas foram importantes para ajudar o desenvolvimento da pesquisa e impulsionar os processos de TT para as empresas. Viginier (2002, p.111) aponta que os principais objetivos da Lei de Pesquisa de 1982 e da Lei de Pesquisa e Inovação de 1999 foram a promoção da pesquisa nas empresas e a TT e transferência de conhecimento dos laboratórios públicos para as empresas, sobretudo as PME.

Ainda segundo Uzunidis (2001, p.168-170), a Lei de 1999 também trouxe novos paradigmas para a realidade francesa. A referida lei permitiu aos pesquisadores criar empresas conservando seus status de funcionários públicos, às universidades criar incubadoras universitárias e também permitiu a criação da SAS (Société par actions simplifiée), além de também estabelecer ao pesquisador público o direito de $50 \%$ sobre os créditos financeiros provenientes de suas invenções.

Em um panorama geral, o impacto das medidas legais sobre o financiamento de pesquisa P\&D entre setor público e empresas foi positivo para as instituições públicas de pesquisa. Segundo Viginier (2002, p. 114), após a Lei de 1999, os recursos recebidos pelas instituições públicas de pesquisa para os contratos de P\&D se elevaram em 2,33milhões de euros, representando $16 \%$ de seus recursos totais contra $10 \%$ no início dos anos noventa.

Além disso, Jacquin (2003, p.102) enfatiza que os efeitos da lei de 1999 foram bastante positivos para a criação de spin-offs a partir da pesquisa pública. O número de empresas criadas de 1999 a 2002 foi de 347, o que representa centenas de empresas por ano.

Para a ULR, talvez esses resultados ainda sejam uma realidade a ser alcançada, principalmente se avaliarmos o pouco tempo de existência da universidade e do seu organismo 
interno de TT, além de considerarmos que o sistema local de inovação ao qual a ULR está atrelada ainda está em processo de desenvolvimento. Entretanto, a ULR tem se mostrado atenta ao movimento do sistema nacional de inovação e às oportunidades de criação de organismos para a TT como o ULR Valor, Fundação de La Rochelle, SATT e Valconum.

Já para a realidade brasileira, no que tange aos dispositivos legais, a década de 1990 representou os primeiros passos da política industrial voltada para inovação. Esses passos foram consequência de debates acerca da importância de investimentos em P\&D nacional para alavancar o índice de inovação no país e, em certa medida, equilibrar a balança de pagamento brasileira (MARCOVITCH, 1980), promovendo maior exportação de produto com maior valor agregado.

Porém apenas em 2004, foi lançada a Lei Federal de Inovação, que segundo Matias-Pereira e Kruglianskas (2005), sofreu uma grande influência da Lei francesa de 1999.

A regulamentação da Lei de Inovação Tecnológica (LIT) implica uma série de mudanças no comportamento das instituições de pesquisa. De acordo com Matias-Pereira e Kruglianskas (2005), abre-se a possibilidade de as instituições científicas e tecnológicas, inclusive as federais de ensino superior, dividirem seus laboratórios, equipamentos, instrumentos, materiais e demais instalações com empresas para desenvolvimento de atividades dirigidas à inovação tecnológica em parceria com o setor privado.

Muito embora tenha ocorrido um avanço no dispositivo legal quanto à regulamentação da política de inovação no Brasil, Matias-Pereira e Kruglianskas (2005) ressaltam que o teor da LIT e de sua regulamentação mostram-se particularmente deficientes nos aspectos que envolvem a flexibilidade de gestão das instituições de pesquisa, bem como nos assuntos relacionados às competências de outros ministérios.

A falta de flexibilidade, a impossibilidade de criação de organismos externos para a gestão da PI ou TT, a ausência de dispositivos que autorizem a criação de fundações universitárias para as universidades estaduais da Bahia, a dificuldade de criação de cargos e funções para os Núcleos de Inovação Tecnológica representam as principais diferenças entre a realidade da ULR e a UESC.

\section{Conclusão}

Conclui-se que tanto a UESC quanto a ULR apresentam reais esforços para desenvolver suas atividades de PI e TT. Ambas possuem em sua essência as funções de ensino, pesquisa e extensão e auxiliam de forma substancial o desenvolvimento sócio econômico das suas respectivas áreas de abrangência.

No que se refere à gestão da Inovação, PI e TT, a principal diferença existente entre as universidades comparadas reside nos dispositivos legais aos quais elas estão submetidas. 
As Leis de Inovação francesa e brasileira possuem em sua essência, o mesmo conteúdo e conferem às universidades os mesmos direitos e praticamente as mesmas possibilidades de atuação.

Entretanto, a ULR ainda goza de outros dispositivos legais que a conferem maior autonomia, flexibilidade e agilidade nos processos de TT e gestão de PI, enquanto que as legislações brasileira e baiana não possibilitam à UESC a criação de uma fundação ou mesmo de um organismo externo à instituição para desenvolver as ações de TT e PI.

Uma solução para essa questão seria a promulgação de leis que conferissem às universidades brasileiras maior autonomia e flexibilidade na gestão de suas PI e nos processos de TT. Assim, elas poderiam alcançar um maior nível de competitividade frente à realidade atual e contribuir de forma mais decisiva ao desenvolvimento socioeconômico do país.

\section{Referências}

ANDREASSI, T. Estudo das relações entre indicadores de P\&D e indicadores de resultado empresarial em empresas brasileiras. 1999. Tese (Doutorado) - Faculdade de Economia, Administração e Contabilidade da Universidade de São Paulo, São Paulo.

BALBINOT, Z., MARQUES, R. A.. Alianças Estratégicas Como Condicionantes Do Desenvolvimento Da Capacidade Tecnológica: O Caso De Cinco Empresas Do Setor EletroEletrônico Brasileiro. Revista de Administração Contemporânea, v.13, n. 4, p.604-625, 2009.

COSTA, P. R. E; PORTO, G. S. Capacitação E Inovação: Aspectos Do Desenvolvimento Interno e Externo nas Multinacionais Brasileiras. REGE - Revista de Gestão, São Paulo, v. 18, n. 3, p. 297 322, Jul./Set. 2011.

DIAS, R. Emulação De Arranjos Institucionais E Foco Na Alta Tecnologia: Inadequação Da Política Científica E Tecnológica No Contexto De Um País Subdesenvolvido. Revista Espaço Acadêmico, n. 73, Ano VII, Junho/2007.

GARNICA, L. A., TORKOMIAN, A. L. V. Gestão de tecnologia em universidades: uma análise do patenteamento e dos fatores de dificuldades e de apoio á transferência de tecnologia no estado de São Paulo. Gestão e Produção, São Carlos, v. 16, n. 4, p. 624-638, 2009.

INPI. Busca de Patentes. Disponível em: http://formulario.inpi.gov.br/MarcaPatente/servlet/PatenteServletController Acesso em: 23 mai. 2013.

JACQUIN, J. Les jeunes etreprises innovantes : une priorité pour la croissance. Paris : La Documentation Française, 2003. 
MALKIN, D. 2005. Table Ronde: Options pour le système français d'innovation. In: LAREDO, P., SACHWALD, F. (Coord.). Le système francais d'innovation dans l'économie mondiale : enjeux et priorités. IFRI, Institut de l'enterprise. p. 107, 2005.

MATIAS-PEREIRA, J., KRUGLIANSKAS, I. Um enfoque sobre a Lei de Inovação Tecnológica do Brasil. RAP, Rio de Janeiro, v. 39, n. 5, p 1011-1029, Set./Out. 2005.

MÉRINDOL, J.-Y.. Table Ronde: Options pour le système français d'innovation. In: LAREDO, P., SACHWALD, F. (Coord.). Le système francais d'innovation dans l'économie mondiale : enjeux et priorités. IFRI, Institut de l'enterprise. p. 108-110, 2005.

SANTANA, E. E. P., PORTO, G. S. E agora, o que fazer com essa tecnologia? Um estudo multicaso sobre a possibilidade de transferência de tecnologia na USP-RP. Revista de Administração Contemporânea, Curitiba, v. 13, n. 3, art. 4, p. 410-429, 2009.

TAKAHASHI, V.P.; SACOMANO, J. B. Proposta De Um Modelo Conceitual Para Análise Do Sucesso De Projetos De Transferência De Tecnologia: Estudo Em Empresas Farmacêuticas. Gestão e Produção, v.9, n.2, p. 181-200, Ago/2002.

TIGRE, P. B. Gestão da Inovação: a economia da tecnologia do Brasil. Rio de Janeiro: Elsevier, 2006.

UNIVERSIDADE ESTADUAL DE SANTA CRUZ. História. Disponível em: http://www.uesc.br/a_uesc/index.php?item=conteudo_historia.php. Acesso em: 25 mai. 2013.

ASPLAN: UESC em dados 2011. Disponível em:

http://www.uesc.br/asplan/dados/uesc2011.pdf. Acesso em: 25 mai. 2013.

NIT: CONSU 05/2009. Disponível em:

http://www.uesc.br/nucleos/nit/consu05.2009_nit.pdf. Acesso em: 25 mai. 2013.

UNIVERSITÉ DE LA ROCHELLE. L'Université d'hier à aujourd'hui. Disponível em: http://www.univ-larochelle.fr/L-Universite-d-hier-a-aujourd-hui. Acesso em: 25 mai. 2013.

Les chiffres clés. Disponível em: http://www.univ-larochelle.fr/Les-chiffres-cles-2013. Acesso em: 25 mai. 2013.

Contrat pluriannuel de développement 2012-2017. Disponível em: http://www.univlarochelle.fr/IMG/pdf/201203_com_ctr12_ulr_vd_270312.pdf. Acesso em: 25 mai. 2013.

UZUNIDIS, D. La marchéisation de la recherche publique en France et la question de la propriété industrielle. In : LAPERCHE, B. (Coord.). Propriété Industrielle et Innovation : La « nouvelle économie » fausse-t-elle l'enjeu ? Paris: L'Harmattan, p. 159-174, 2001.

URL VALOR. Qui sommes nous. Disponível em: http://ulrvalor.univ-larochelle.fr/-Qui-sommesnous- Acesso em: 25 mai. 2013. 
Société de droit prive. Disponível em: http://ulrvalor.univ-larochelle.fr/article/societede-droit-prive. Acesso em: 25 mai. 2013.

VALCONUM. Historique. Disponível em: http://www.valconum.fr/. Acesso em: 25 mai. 2013.

Membres. Disponível em: http://www.valconum.fr/. Acesso em: 25 mai. 2013.

VARGAS, J. I. Alguns Aspectos da Política Nacional de Ciência e Tecnologia. Química Nova, v. 20 (Especial), 1997.

VIGINIER, P. La France dans l'économie du savoir : pour une dynamique collective. Paris: La Documentation Française, 2002.

Inserir aqui dados completos de TODOS os autores:

Nome completo: Fabiana Carneiro Silva de Holanda

Filiação institucional: Universidade Estadual de Santa Cruz

Departamento: Agroindústria

Função ou cargo ocupado: Agente de Inovação

Endereço completo para correspondência (bairro, cidade, estado, país e CEP): Rodovia Jorge

Amado, KM 16 Ilhéus/BA - Brasil 45662-900

Telefones para contato: 73 - 36805190

e-mail: fabianaholanda2010@hotmail.com

Nome completo: Gesil Sampaio Amarante Segundo

Filiação institucional: Universidade Estadual de Santa Cruz

Departamento: Núcleo de Inovação Tecnológica

Função ou cargo ocupado: Coordenador

Endereço completo para correspondência (bairro, cidade, estado, país e CEP): Rodovia Jorge

Amado, KM 16 Ilhéus/BA - Brasil 45662-900

Telefones para contato: 73 - 36805190

e-mail: gesil.amarante@gmail.com

Nome completo: Mickaël Coustaty

Filiação institucional: Université de La Rochelle

Departamento:

Função ou cargo ocupado:

Endereço completo para correspondência (bairro, cidade, estado, país e CEP): CCA, bureau 19 - 5, allée de l'Océan 17000 La Rochelle - France

Telefones para contato: 330546458763

e-mail:m.coustaty@valconum.fr

Nome completo: Eric de Almeida Monteiro

Filiação institucional: Université de La Rochelle

Departamento: Lettres, Langues, Arts Et Sciences Humaines

Função ou cargo ocupado: Professor

Endereço completo para correspondência (bairro, cidade, estado, país e CEP): Technoforum I 23, avenue Albert Einstein, 17071 La Rochelle - Cedex 9 - France

Telefones para contato: +33 5.46.45.68.61

e-mail: eric.monteiro@univ-lr.fr 